\title{
BARREIRAS PARA ADOÇÃO DE FERRAMENTAS EDUCACIONAIS BASEADAS EM REALIDADE AUMENTADA: UMA REVISÃO DA LITERATURA
}

Suédia Rosane de Lima Sales Souza1; Ingrid Winkler²; Sayonara Nobre de Brito Lordelo ${ }^{3}$

'Universitário Senai Cimatec, Av. Orlando Gomes, 1845 - Piatã, Salvador/ BA, 41650 010; Suédia Rosane de Lima Sales Souza-suedialima@hotmail.com

Resumo: A educação está em constante mudança, na tentativa de agregar ao processo de ensino-aprendizagem aspectos que contribuam para uma educação de qualidade. Neste contexto, este estudo teve por objetivo caracterizar a produção científica sobre as barreiras para adoção de ferramentas educacionais em Realidade Aumentada. A partir de uma revisão da literatura, foram inicialmente selecionados 44 trabalhos nas bases científicas Science Direct e Scielo. As barreiras foram classificadas em nove categorias: técnica, financeira, conteúdo, aprendizado, segurança, adesão, interação com o usuário, visualização e período. Apenas 17 estudos apresentam resultado mensurável. Conclui-se que são necessários mais estudos quanto às barreiras para adoção desta tecnologia no domínio da educação.

Palavras-Chave: Realidade Aumentada; Educação; Barreiras; Adoção.

\section{BARRIERS FOR ADOPTION OF EDUCATIONAL TOOLS BASED ON INCREASED REALITY: A LITERATURE REVIEW}

\begin{abstract}
Education is constantly changing in an attempt to add to the teachinglearning process aspects that contribute to quality education. In this context, this study aimed to characterize the scientific production on the barriers to the adoption of augmented reality educational tools. From a literature review, 44 studies were initially selected from the Science Direct and Scielo scientific bases. Barriers were classified into nine categories: technical, financial, content, learning, security, adherence, user interaction, visualization, and time period. Only 17 studies have measurable results. It is concluded that further studies are needed regarding the barriers to the adoption of this technology in the field of education.
\end{abstract}

Keywords: Augmented Reality; Education; Barriers; Adoption 


\section{INTRODUÇÃO}

O uso de novas tecnologias tem proporcionado avanços e aprimorado os métodos tradicionais de ensino, facilitando o aprendizado por parte dos alunos e também alterando a forma como o professor planeja a construção do conhecimento, por isso a tecnologia está cada vez mais presente no processo de ensinoaprendizagem. É importante ressaltar que as ferramentas digitais, não trazem benefícios, apenas por seu uso, é preciso que haja uma intencionalidade, observar o emprego da tecnologia como uma importante ferramenta nos processos de ensinar e aprender e não considerar seu uso com um fim em si mesmo. Pois, como afirma o autor [1], percebe-se que "[...] nas condições de verdadeira aprendizagem, os educandos vão se transformando em reais sujeitos da construção e da reconstrução do saber ensinado [...]". Isso potencializará o percurso norteador do processo de ensino-aprendizagem, melhorando os indicadores de aprendizagem dos alunos, a participação em sala de aula, despertando o aluno para a criação de novos saberes.

A Realidade Aumentada (RA) foi introduzida pela primeira vez como uma ferramenta de treinamento para pilotos de linhas aéreas e da Força Aérea durante os anos 90 [2]. Segundo a definição clássica de [3], a RA é um sistema que complementa o mundo real com objetos virtuais (gerados por computador) que parecem coexistir no mesmo espaço que o mundo real. [4] afirmam que uma das razões mais importantes pelas quais essa tecnologia é tão usada é que ela não precisa mais de um hardware caro e equipamento sofisticado. Porém, os estudos recentes apontam que ainda poucas instituições de ensino adotam a tecnologia de RA, no processo de ensino aprendizagem.

Este estudo teve por objetivo caracterizar a produção científica atual sobre as barreiras para adoção de ferramentas educacionais baseadas em Realidade Aumentada. $O$ trabalho está organizado em quatro seções: além desta Introdução, a seção 2 descreve a metodologia utilizada na investigação; a seção 3 aborda os resultados e discute-os, na seção 4 a conclusão.

\section{METODOLOGIA}

Quanto aos procedimentos metodológicos, a pesquisa caracteriza-se como um estudo qualitativo, de caráter exploratório, a partir de uma revisão da literatura, que tem como meta, proporcionar maior familiaridade com o problema, no qual, busca-se identificar as barreiras, dificuldades e desafios para disseminação de ferramentas educacionais da tecnologia RA.

Esta pesquisa, analisou os estudos realizados no período entre 2014 a 2019 , em duas bases de dados: Science Direct e Scielo, e teve como software de gerenciamento de referências Mendeley. Para realização da pesquisa, foram utilizados os descritores de busca ("augmented reality" OR "mixed reality" OR "extended reality") AND (education OR learning) AND (barrier OR challenge OR difficulties). A string de busca foi aplicada somente nos campos de palavras-chave, título e resumo. 
A pesquisa foi realizada a partir dos critérios de exclusão (CE) e inclusão $(\mathrm{Cl})$. Para o CE trabalhos que não tiveram metodologia bem clara e trabalhos que não avaliaram as barreiras para a adoção de ferramentas educacionais em RA, foram excluídos. Para o critério $\mathrm{Cl}$, foram selecionados trabalhos que trataram das barreiras para adoção de ferramentas educacionais em RA, que tiveram foco nas barreiras que inibissem a difusão de ferramentas educacionais de RA e trabalhos recentes, que já possuam aprovação pela comunidade científica. Manteve-se apenas as publicações relevantes para essa revisão. Os trabalhos selecionados foram artigos de revisão e artigos de pesquisa. Dos 44 estudos encontrados, a amostra foi reduzida para 17 artigos. A Figura 1, ilustra os critérios adotados até o resultado já posto, a serem analisados e discutidos nesta revisão integrativa.

Figura 1. Processo de Seleção das Publicações

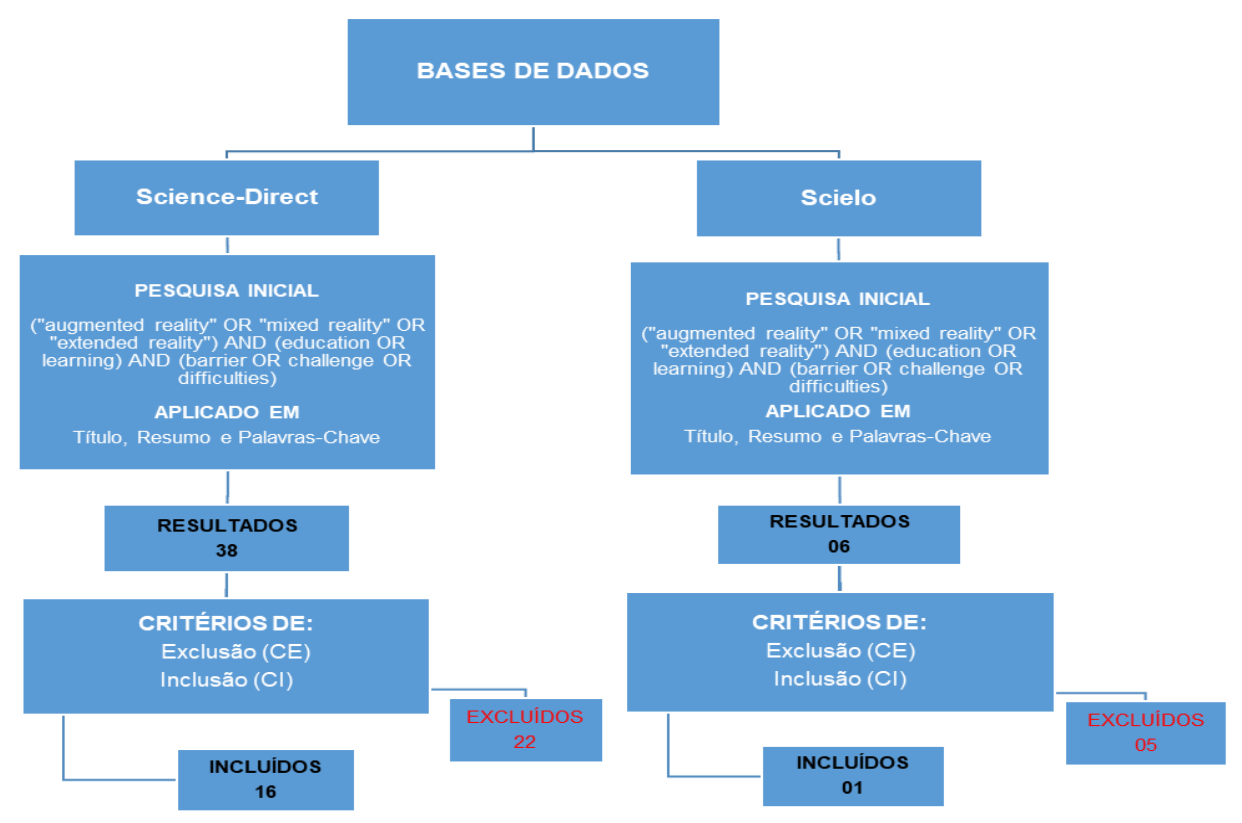

\section{RESULTADOS E DISCUSSÃO}

Dos estudos da amostragem, 17 artigos apresentaram, pelo menos, um indicador correspondente as barreiras para adoção de ferramentas educacionais, baseadas em Realidade Aumentada. Entre as categorias apresentadas na análise, aparecem mais de uma categoria por autor, sendo algumas citadas por mais de um autor. As barreiras, dificuldades e desafios identificados foram agrupadas em nove categorias:
1) Técnica
2) Financeira
3) Conteúdo
4) Aprendizado
5) Segurança
6) Adesão
7) Interação com o usuário
8) Visualização
9) Período 


\section{\begin{tabular}{l|l} 
CIRCULAR ECONOMY & ECONOMIA CIRCULAR
\end{tabular}}

Tabela 1. Classificação das barreiras em categorias

\begin{tabular}{|c|c|c|}
\hline CATEGORIAS & ARTIGOS & BARREIRAS \\
\hline TÉCNICA & {$[4,5,6,7,8,9]$} & $\begin{array}{l}\text { Instabilidade da ferramenta RA durante o uso; softwares com baixa capacidade; } \\
\text { problemas relacionados à conexão entre tecnologias e formatos; problemas com } \\
\text { os tamanhos e sistemas de visualização; problemas técnicos; baixa conexão com } \\
\text { a Internet; limitações tecnológicas, dispositivos que não são compatíveis; alto } \\
\text { consumo de bateria. }\end{array}$ \\
\hline FINANCEIRA & [4] & $\begin{array}{l}\text { Dificuldades relacionadas a alto custo de aquisição ou desenvolvimento de } \\
\text { ferramentas }\end{array}$ \\
\hline CONTÉUDO & {$[9,18]$} & $\begin{array}{l}\text { Falta de habilidades de programação; dificuldade de desenvolvimento das } \\
\text { atividades utilizando Realidade Aumentada. }\end{array}$ \\
\hline APRENDIZADO & {$[4,21,22]$} & $\begin{array}{l}\text { Poucas experiências com a tecnologia Realidade Aumentada na educação; } \\
\text { poucos estudos sobre as opiniões dos professores para verificar a sua percepça } \\
\text { do valor que a RA pode ter no processo de ensino e aprendizagem; poucos } \\
\text { estudos abordam, sobrecarga cognitiva que a RA pode gerar em um ambiente de } \\
\text { aprendizagem. }\end{array}$ \\
\hline SEGURANÇA & [9] & Problemas de segurança e privacidade dos dados. \\
\hline ADESÃO & $\begin{array}{l}{[4,6,7,9,10,11} \\
12,13]\end{array}$ & $\begin{array}{l}\text { Tecnologias de RA em estado embrionário em aplicação na educação; dificuldades } \\
\text { na aceitação dos usuários; dificuldade de usar a geração de conteúdo dos } \\
\text { dispositivos moveis; necessidadde de restruturar o setor pedagógico; poucos } \\
\text { trabalhos foram realizados para fundamentar que a RA é uma tecnologia com } \\
\text { grande potencial para impactar os resultados de aprendizagem afetivos e } \\
\text { cognitivos; poucos estudos sobre amostras de dados para identificar as causas da } \\
\text { eficácia da aprendizagem do aplicativo baseado em RA. }\end{array}$ \\
\hline $\begin{array}{l}\text { INTERAÇÃO COM } \\
\text { O USUÁRIO }\end{array}$ & $\begin{array}{l}{[4,5,6,9,10,14} \\
15,16]\end{array}$ & $\begin{array}{l}\text { Poucos estudos sobre o efeito do método cognitivo que a ferramenta RA fornece; } \\
\text { dificuldade cognitiva; é preciso estimular mas ambientes com uso da tecnologia } \\
\text { RA; poucas bases tem materiais de visualização com mais opções em } \\
\text { plataformas multimídia; pouca cooperação entre professores e desenvolvedores na } \\
\text { ferramentas de RA; dificuldades na usabilidade dos recursos de RA por alunos e } \\
\text { professores; problemas de usabilidade. }\end{array}$ \\
\hline VISUALIZAÇÃO & {$[10,14]$} & $\begin{array}{l}\text { Dificuldade no processamento em imagem dinâmica; baixa qualidade de brilho das } \\
\text { tecnologias RA. }\end{array}$ \\
\hline PERÍODO & {$[4,19]$} & $\begin{array}{l}\text { Pouco tempo de aula para implementar alguns aplicativos de Realidade } \\
\text { Aumentada. }\end{array}$ \\
\hline
\end{tabular}

\subsection{Categoria Técnica, Financeira e Segurança}

A categoria Técnica, apareceu em $54 \%$ dos artigos analisados, muitos estudos $[4,5,6,7,8]$ destacam as seguintes barreiras e dificuldades: Instabilidade da ferramenta RA durante o uso; softwares com baixa capacidade; poucos sistemas concentrados em ensinar como executar as tarefas; problemas técnicos em aplicações de RA baseadas em localização; problemas técnicos, como baixa sensibilidade, disparam reconhecimento e erro GPS; baixa conexão com a internet. A manutenção de informações sobrepostas é uma limitação do sistema de RA; no estudo de [9], também mostra que a falta de suporte técnico é uma barreira que impacta a implementação da RA, como limitações tecnológicas, dispositivos que não são compatíveis; alto consumo de bateria com o uso das aplicações, dificuldade de algumas aplicações no uso mais amplo em ambientes livres, sem uso de marcadores. 


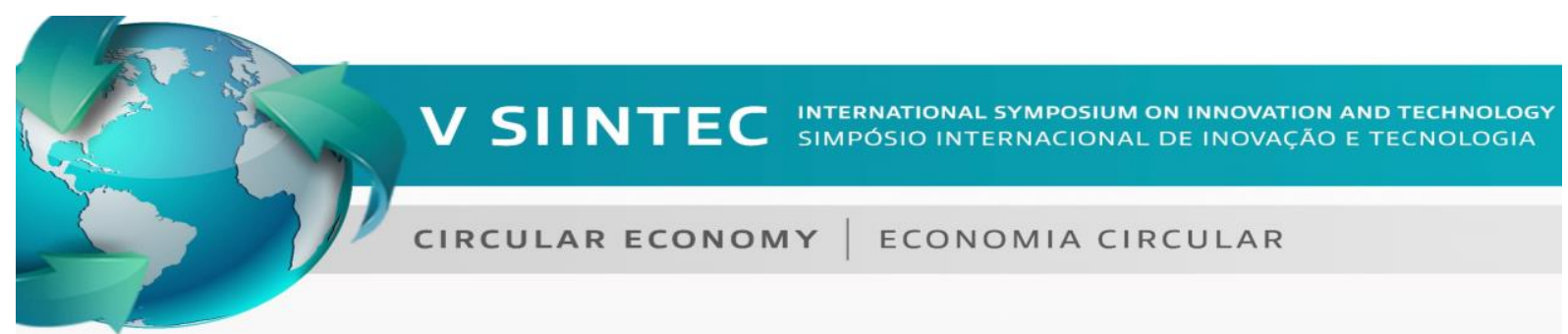

Tendo em vista os resultados obtidos, as categorias menos abordadas, foram Financeira e Segurança com 9\%, dos artigos analisados. No estudo de [4], a categoria Financeira apresentou dificuldade do uso da tecnologia RA com grandes grupos, podendo gerar um custo proibitivo. Já na categoria Segurança, o autor [9] destaca, problemas de segurança e privacidade, pois com o uso da RA podem ser geradas várias fontes potenciais de invasão, incluindo identificação pessoal, rastreamento de localização e armazenamento de dados privados.

\subsection{Categorias Adesão e Interação com o usuário}

As categorias Adesão e Interação com o usuário, foram a mais citadas com $72 \%$. Na categoria Adesão, o artigo [10] destaca que os desafios que hoje se colocam como métodos de ensino, aquisição e posterior consolidação do conhecimento podem ser atendidos, até certo ponto, pela aplicação de tecnologias. No estudo de [11], também mostra dentre as limitações, a indisponibilidade de literatura relevante sobre o uso da RA na educação; poucas instituições estão determinadas a equipar as próximas gerações com as habilidades relacionadas a capturar, analisar e comunicar dados através dessas infraestruturas de TI. Outros estudos $[6,7,4,12,13]$ apontam barreiras e dificuldades no processo de Adesão da tecnologia, pouco foi feito em termos de aplicações de RA, bem como, o seu impacto numa grande variedade de necessidades educacionais. $\mathrm{Na}$ análise dos dados são apontadas: dificuldades na aceitação dos usuários; dificuldade na diversidade de dispositivos e nos usos comuns de dispositivos móveis, esse por sua vez, é um fator complexo, que sugere dificuldade na implementação de modelos; necessidade de restruturação do setor pedagógico; inadequação em salas de aula lotadas. Segundo o autor [9] é preciso aumentar a aceitação social e muitas aplicações são protótipos ou estão em estágios iniciais.

Já na categoria Interação com o usuário, os autores $[4,5,6,9,10,14,15$, 16], citam que são necessário mais estudos que abordem sobre o efeito do método cognitivo que a ferramenta RA fornece; é preciso estimular mas ambientes de interação entre alunos e professores com uso da tecnologia RA; poucas bases tem materiais de visualização com mais opções em plataformas multimídia usando som ou vídeo; outro ponto observado, é a necessidade de aumentar a cooperação entre professores e desenvolvedores para especificar a quantidade e o tipo de informações que devem ser colocadas na ferramentas de RA; possíveis dificuldades na usabilidade dos recursos de RA por alunos e professores e integração ineficaz entre estudantes e professores com o uso da tecnologia. No trabalho de [17], destaca que as principais dificuldades da literatura recente, sobre RA e educação, nas quais são orientados a deixar sempre como ponto de partida um apoio pedagógico claro e forte, que garante, que os processos beneficiem os alunos e não que eles permaneçam em mais uma novidade.

\subsection{Categorias Conteúdo, Visualização, Período e Aprendizado}


As categorias Conteúdo, Visualização e Período foram identificadas em 18\% dos artigos. Na categoria Conteúdo o autor [18], destacou as seguintes barreiras e dificuldades: falta de habilidades de programação, como sendo uma barreira para o engajamento dos professores no desenvolvimento e customização de suas próprias aplicações; no estudo [9], dificuldade de desenvolvimento das atividades utilizando RA, principalmente por parte dos professores que não dominam o uso dos softwares e dos equipamentos para o desenvolvimento das aplicações. Já na categoria Visualização, foram identificadas: dificuldade no processamento em imagem dinâmica; e necessidade de melhorar as condições de brilho das tecnologias RA. Na categoria Período, os estudos apresentaram restrições de tempo para a aprendizagem, [19] cita que é preciso investir um tempo maior em explicação, para os alunos sobre como usar a tecnologia, sessões de aulas normais não fornecem tempo suficiente para implementar alguns aplicativos de RA. No estudo de [20], contribui relatando que alguns educadores não fazem o uso da tecnologia por que se sentem incapazes ou não querem continuar usando-os, devido a uma série de problemas, em diversas áreas, incluindo suporte técnico, atitudes de estudantes e pressões de tempo.

A categoria Aprendizado, corresponde a $27 \%$ dos estudos. Em três estudos $[4,21,22]$ traz que as expectativas referidas as estratégias mais adequadas para 0 uso da tecnologia no ambiente de aprendizado, precisam ser validados e mais explorados, o desafio não é apenas a introdução de tecnologia na sala de aula, mas principalmente como usá-la para melhorar aprendizado do aluno, as experiências ainda estão em seus estágios iniciais, os alunos não têm experiência prévia com sistemas de RA e a maioria deles nem sequer conhece este tipo de tecnologia. Outras barreiras identificadas foram os efeitos colaterais da RA, tais como: esforço mental ou ansiedade de aprendizagem; dificuldade dos alunos no uso da RA, sobrecarga cognitiva dos estudantes, a maioria dos outros desafios relatados envolve problemas técnicos e relacionados a aplicativos. O artigo [23], contribui afirmando que, o papel importante que os professores têm na adoção de tecnologia nas escolas, defende o envolvimento de professores na avaliação de formas mais ativas quanto possível.

\section{CONCLUSÃO}

Este trabalho teve como objetivo caracterizar a produção científica atual sobre as barreiras para adoção de ferramentas educacionais em RA. Para tanto, dos estudos da amostragem, 17 apresentaram, pelo menos, um indicador correspondente. As barreiras mais citadas, foram Adesão e Interação com o usuário com $72 \%$, em seguida Técnica, que apareceu em $54 \%$, dos trabalhos, demostrando, maior índice de dificuldades e barreiras existentes no processo de implementação da tecnologia Realidade Aumentada na aprendizagem.

Os resultados parciais obtidos nesta SLR, revelaram que ainda são, consideravelmente, poucos os estudos acerca dessa temática. Vale ressaltar, que está SLR levou em consideração apenas a base de dados Science-Direct, Scielo e o próximo passo é ampliar a investigação para base de dado Scopus, e consequentemente expande as análises. No entanto, mesmo como resultados 
preliminares, espera-se que a análise das barreiras e desafios para adoção de ferramentas educacionais da tecnologia RA, tratada nesse estudo, possibilite aos futuros pesquisadores dessa temática, um embasamento norteador para suas pesquisas.

\section{REFERÊNCIAS}

${ }^{1}$ FREIRE, P. 1996. Pedagogia da autonomia: saberes necessários à prática.

${ }^{2}$ CAUDELL, T.P., \& MIZELL, D. W. 1992. Augmented reality: na application pf headsup display technology to manual manufacturing processes. In proceedings of the twenty-fifth hawaii international conference on system sciences.

${ }^{3}$ AZUMA, RONALD et al. 2018. Recente advances in augmented reality. IEEE computer graphics and applications, V. 21, N. 6, P. 34-47.

${ }^{4}$ AKC AYIR, Murat; AKC AYIR, Gökçe. Advantages and challenges associated with augmented reality for education: A systematic review of the literature. educational research review, v. 20, p. 1-11, 2017.

${ }^{5} \mathrm{CAI}, \mathrm{Su}$; WANG, Xu; CHIANG, Feng-Kuang. A case study of Augmented Reality simulation system application in a chemistry course. Computers in human behavior, v. 37, p. 31-40, 2014.

${ }^{6} \mathrm{BACCA}$, Jorge et al. Mobile augmented reality in vocational education and training. procedia Computer Science, v. 75, p. 49-58, 2015.

${ }^{7}$ FONSECA, David et al. Relationship between student profile, tool use, participation, and academic performance with the use of Augmented Reality technology for visualized architecture models. Computers in human behavior, v. 31, p. 434-445, 2014.

${ }^{8}$ GARZÓN, Juan; ACEVEDO, Juan. A Meta-analysis of the impact of Augmented Reality on students' learning effectiveness. Educational Research Review, 2019.

9 LOPES, Luana M. D. et al. Inovações Educacionais com o uso da Realidade Aumentada: uma revisão sistemática. Educação em Revista, v. 35, n. 1, 2019.

${ }^{10}$ COIMBRA, MMath Teresa; CARDOSO, Teresa; MATEUS, Artur. Augmented reality: an enhancer for higher education students in math's learning?. Procedia Computer Science, v. 67, p. 332-339, 2015. 
${ }^{11}$ ELLAHI, Rizwan Matloob; KHAN, Moin Uddin Ali; SHAH, Adeel. Redesigning Curriculum in line with Industry 4.0. Procedia Computer Science, v. 151, p. 699708, 2019.

${ }^{12}$ IBÁÑEZ, María Blanca et al. Experimenting with electromagnetism using augmented reality: Impact on flow student experience and educational effectiveness. Computers \& Education, v. 71, p. 1-13, 2014.

13TURKAN, Yelda et al. Mobile augmented reality for teaching structural analysis. Advanced Engineering Informatics, v. 34, p. 90-100, 2017.

${ }^{14}$ JEŘÁBEK, Tomáš; RAMBOUSEK, Vladimír; WILDOVÁ, Radka. Specifics of visual perception of the augmented reality in the context of education. Procedia-Social and Behavioral Sciences, v. 159, p. 598-604, 2014.

${ }^{15}$ KURNIAWAN, Michael $\mathrm{H}$. et al. Human anatomy learning systems using augmented reality on mobile application. Procedia Computer Science, v. 135, p. 80-88, 2018.

${ }^{16}$ LEE, Hyunjeong et al. Cooperation begins: Encouraging critical thinking skills through cooperative reciprocity using a mobile learning game. Computers \& Education, v. 97, p. 97-115, 2016.

${ }^{17}$ CUPITRA-GARCÍA, Alberto; DUQUE-BEDOYA, Erika Teresa. Profesores aumentados en el contexto de la realidad aumentada: una reflexión sobre su uso pedagógico. El Ágora USB, v. 18, n. 1, p. 245-255, 2018.

${ }^{18}$ MOTA, José Miguel et al. Augmented reality mobile app development for all. Computers \& Electrical Engineering, v. 65, p. 250-260, 2018.

${ }^{19}$ LARA-PRIETO, Vianney et al. An innovative self-learning approach to 3D printing using multimedia and augmented reality on mobile devices. Procedia computer science, v. 75, p. 59-65, 2015.

${ }^{20}$ GREGORY, SUE et al. 2015. Barriers and enablers to the use of virtual worlds in higher education: na exploration of educator perceptions, atitudes and experiences. Jornal of educational technology \& society, V. 18, N.1, P. 3-12.

${ }^{21}$ SAMPAIO, Daniel; ALMEIDA, Pedro. Pedagogical strategies for the integration of Augmented Reality in ICT teaching and learning processes. Procedia Computer Science, v. 100, p. 894-899, 2016.

${ }^{22} \mathrm{HSU}$, Ting-Chia. Learning English with augmented reality: Do learning styles matter?. Computers \& Education, v. 106, p. 137-149, 2017.

${ }^{23}$ DA SILVA, Manoela MO et al. 2019. Perspectives on how to evaluate augmented reality technology tools for education: a systematic review. jornal review. jornal of the brszilian computer society, V. 25, N. 1, P. 3. 